\title{
ANALYSIS OF POLE POSITION FROM 1846 TO 1970*
}

\author{
E. M. GAPOSCHKIN \\ Smithsonian Astrophysical Observatory, Cambridge, Mass. 02138, U.S.A.
}

\begin{abstract}
One- and two-component models for the Chandler motion are investigated with the use of historical data. Evidence for a two-component motion is more convincing from both the dataanalysis and the geophysical point of view.
\end{abstract}

\section{Introduction}

The discussion of polar motion in classical texts (e.g., see Munk and MacDonald, 1960; Jeffreys, 1970) assumes a model for the motion consisting of an elastic Earth with damping and oceans, a system with one degree of freedom. In the literature, analyses of the data frequently indicate that a more complicated motion actually exists. Such models have been discarded owing to 'unreasonableness', yet retention of a one-parameter model leads to abnormally high dissipation and to other consequences that can be viewed sceptically. In this analysis, we study historical data on pole position and investigate the consequences.

There is general agreement on the component with a 365.25 -day period. It is a forced motion due to meteorological effects, and the determinations of it are all consistent. The remaining motion is ascribed to the free nutation of an elastic earth and is called the Chandler motion.

Analyses of polar-motion data can be divided into two groups: those assuming a one-parameter system, seeking a natural frequency for the nutation (summarized in Table I) and those characterizing the motion in a more intricate way (summarized in Table II).

TABLE I

One-parameter systems

\begin{tabular}{ll}
\hline Reference & Period (days) \\
\hline Chandler (1891) & 428. \\
Jeffreys (1940) & 439. \\
Rudnick (1956) & 435.75 \\
& 432.1 \\
Walker and Young (1957) & various values between \\
Munk and MacDonald (1960) & $430.6-470.1$ \\
Jeffreys (1968) & 430.3 \\
\hline
\end{tabular}

* This work was supported in part by grants NGR 09-015-002 and NGR 09-015-103 from the National Aeronautics and Space Administration. 
TABLE II

Complex systems

\begin{tabular}{ll}
\hline Reference & Interpretation \\
\hline $\begin{array}{l}\text { Labrouste and Labrouste (1946) (as } \\
\text { mentioned in Coulomb and Jobert, }\end{array}$ & $\begin{array}{l}\text { Four uniform oscillations with periods of } \\
405,427,441, \text { and } 457 \text { days }\end{array}$ \\
$\begin{array}{l}\text { R63) } \\
\text { Rudnick (1956) }\end{array}$ & $\begin{array}{l}\text { Eight frequencies close to the Chandler fre- } \\
\text { quency } \\
\text { Continuous variation between } 414 \text { and } 440 \\
\text { days }\end{array}$ \\
Yelchior (1957) & $\begin{array}{l}\text { Four oscillations with periods of 427, 438, } \\
452, \text { and } 455 \text { days }\end{array}$
\end{tabular}

Melchior's (1957) analysis has been criticized by Munk and MacDonald (1960, pp. 150-153) as involving unreasonable and unobserved changes in physical properties. Yaskov's (1965) analysis has been criticized by Federov and Yatskiv (1965), who invoked a change in phase of $180^{\circ}$, owing to an unknown source, in 1925 . Nesterov and Rykhlova (1970), from a rediscussion of the data, conclude that Melchior's timevariable model is not appropriate.

On the other hand, the single-parameter models result in a very high damping factor or low $Q$ - between 10 and 30. No other determination of $Q$ for the Earth indicates such a small value (Kaula, 1968, p. 99). The bulk of the evidence indicates that $Q$ is independent of frequency and is observed to be between 100 and 1000 for seismic waves and free oscillations. In addition, Melchior (1955), using the analysis of the forced annual terms, and Munk and MacDonald (1966, p. 162), using the equilibrium pole tide, find that $Q<100$ is more appropriate. The low $Q$ results from interpreting the broad spectral response as a one-parameter system. For this reason, Gutenberg (1959, pp. 167-169) follows the findings of Melchior.

It is in this framework that the following analysis is made.

\section{The Data}

There has been general concern about the consistency of the data. Although the data are of remarkable accuracy, the numerous differences in reduction techniques and observing programs have unavoidably caused doubt about the results. For example, one of the critical questions in the discussion will concern the transition of data from the period before 1924 to the subsequent period. Both Federov (1963) and Orlov (1942) discuss in some detail the difficulties of the transition. Yet a critical question raised by Federov and Yatskiv (1965) is whether this transition is, in fact, valid.

Table III lists the data used in this analysis. The data before 1891.5 were read from the curves published by Rykhlova (1971) and are sparse and less accurate than the later data. The other data, though not uniform, are significantly better. These sources are not unique; for example, there is the recent publication by Nicolini (1968). 
TABLE III

Sources of data used in the analysis

\begin{tabular}{lc} 
Reference & Inclusive dates \\
\hline Rykhlova (1967) & $1846.0-1891.5$ \\
Orlov (1942) & $1891.5-1940.0$ \\
Carnera (1948) & $1940.1-1945.9$ \\
Carnera (1949) & $1946.0-1948.8$ \\
Cecchini (1951) & $1948.9-1950.9$ \\
International Astronomical Union (1955) & $1951.0-1954.9$ \\
Cecchini (1962) & $1955.0-1962.0$ \\
Yumi (1964) & $1962.0-1963.0$ \\
Yumi (1965) & $1963.0-1964.0$ \\
Yumi (1966) & $1964.0-1965.0$ \\
IPMS (1971) & $1965.0-1971.0$ \\
\hline
\end{tabular}

It must be understood that in adopting the data from these sources, certain inconsistencies exist and the total data set cannot be considered uniform. For example, the data of Orlov (1942) have been smoothed to remove any secular effect; the more recent data have not. It should also be understood that considerable effort is required to treat the data appropriately, but this effort is well worth while. The consistency of the results obtained here is good enough for us to have confidence in the data.

\section{Analytical Tools}

The analysis is done in two stages. The first is generalized harmonic analysis on the separate components. This has the advantage of dealing with arbitrarily spaced data. We separate the two components and obtain a further check by comparing the phase and amplitude of the components. The second is to combine the components into a complex number $(x+i y)$ and take its Fourier transform. In this case we deal with all the data, and with the fast Fourier transform (FFT), we can economically map the time domain to the frequency domain. We also have analytical relations for the FFT of an exponential function that allow a least-squares adjustment for frequency and amplitude parameters.

\section{A. THE HARMONIC ANALYSIS}

If we let the observation of the pole position $x$ and $y$ at time $t$ be indexed as $x_{j}, y_{j}, t_{j}$, then the amplitude of the $x$ component for some period $P$ is

where

$$
A_{P}^{2}=\left[\frac{\sum_{j} x_{j} \sin (\alpha j)}{\sum_{j} \sin ^{2}(\alpha j)}\right]^{2}+\left[\frac{\sum_{j} x_{j} \cos (\alpha j)}{\sum_{j} \cos ^{2}(\alpha j)}\right]^{2},
$$

$$
\alpha=\frac{2 \pi}{P}\left(t_{j}-T_{0}\right),
$$

and similarly for the $y$ component. 
B. THE DISCRETE FOURIER TRANSFORM (DFT)

The DFT requires equally spaced data points:

$$
\mathbf{x}_{j}=x_{j}+i y_{j},
$$

where the $\mathbf{x}$ denotes a complex number. It is a mapping

$$
\mathbf{X}_{k}=\sum_{j=0}^{N-1} \mathbf{x}_{j} \exp \left(-2 \pi i \frac{j k}{N}\right)
$$

The FFT is an economical method of calculating the array $\mathbf{X}_{k}$, given $\mathbf{x}_{j}$, and requires $N$ to be a power of 2 . Generally this is not available, and we have values for $\mathbf{x}_{j}, j=0, \ldots, M-1$. The DFT is defined by setting

$$
\mathbf{x}_{j}=0 m \leqslant j<N, \quad \mathbf{x}_{j}=\mathbf{A} \exp (2 \pi i f j) .
$$

We can write

$$
\mathbf{X}_{k}=\mathbf{A} \frac{1-\exp \left[2 \pi i\left(f-\frac{k}{N}\right) M\right]}{1-\exp \left[2 \pi i\left(f-\frac{k}{N}\right)\right]}
$$

which allows direct interpretation of the FFT. We use a spectral filter due to Hann, which causes the spurious side bands due to the finite data sample for the raw DFT to decay as $\left(f-f_{0}\right)^{-3}$ rather than as $\left(f-f_{0}\right)^{-1}$. This filter is used by multiplying the data with

$$
\frac{1}{2}\left(1-\cos 2 \pi \frac{j}{M}\right) \text {. }
$$

\section{Analysis of Data from 1891.5 to 1970}

The first operation in analyzing the data is to fit a linear function of time to the data for $x$ and $y$. This function is subtracted from the observations to produce the data for the harmonic analysis. The midpoint used is MJD 25877 (September 22, 1929). The secular motions for $x$ and $y$ are

$$
\begin{aligned}
& x=0.01688+0.0000018095(t-25877) \\
& y=0.056299+0.0000094765(t-25877),
\end{aligned}
$$

with $t$ in days, or

$$
x=1.71+6.68(T) f t \quad y=5.69+35.00(T) f t,
$$

with $T$ in centuries from September 23, 1929.

Markowitz (1968), long an advocate of the secular drift of the pole, found a motion of $0.88 \times 10^{-5}$ per day along a meridian $60^{\circ} \mathrm{W}$. The results given here indicate a drift of $0.96 \times 10^{-5}$ per day along a meridian $73^{\circ} \mathrm{W}$. This analysis is insufficient to prove such drift. Had the Orlov smoothing been used on the whole data set, no secular term would have been found. 


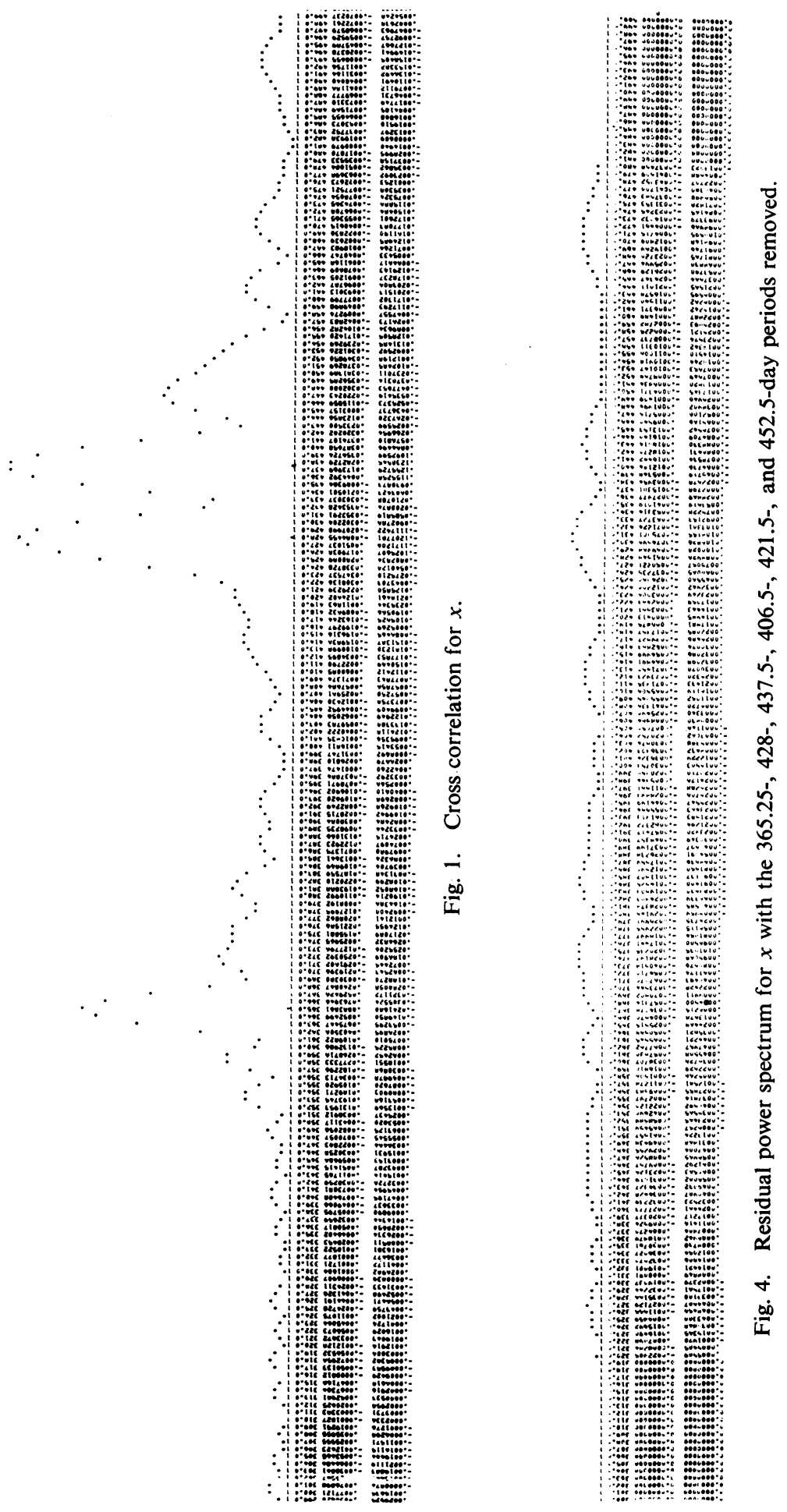



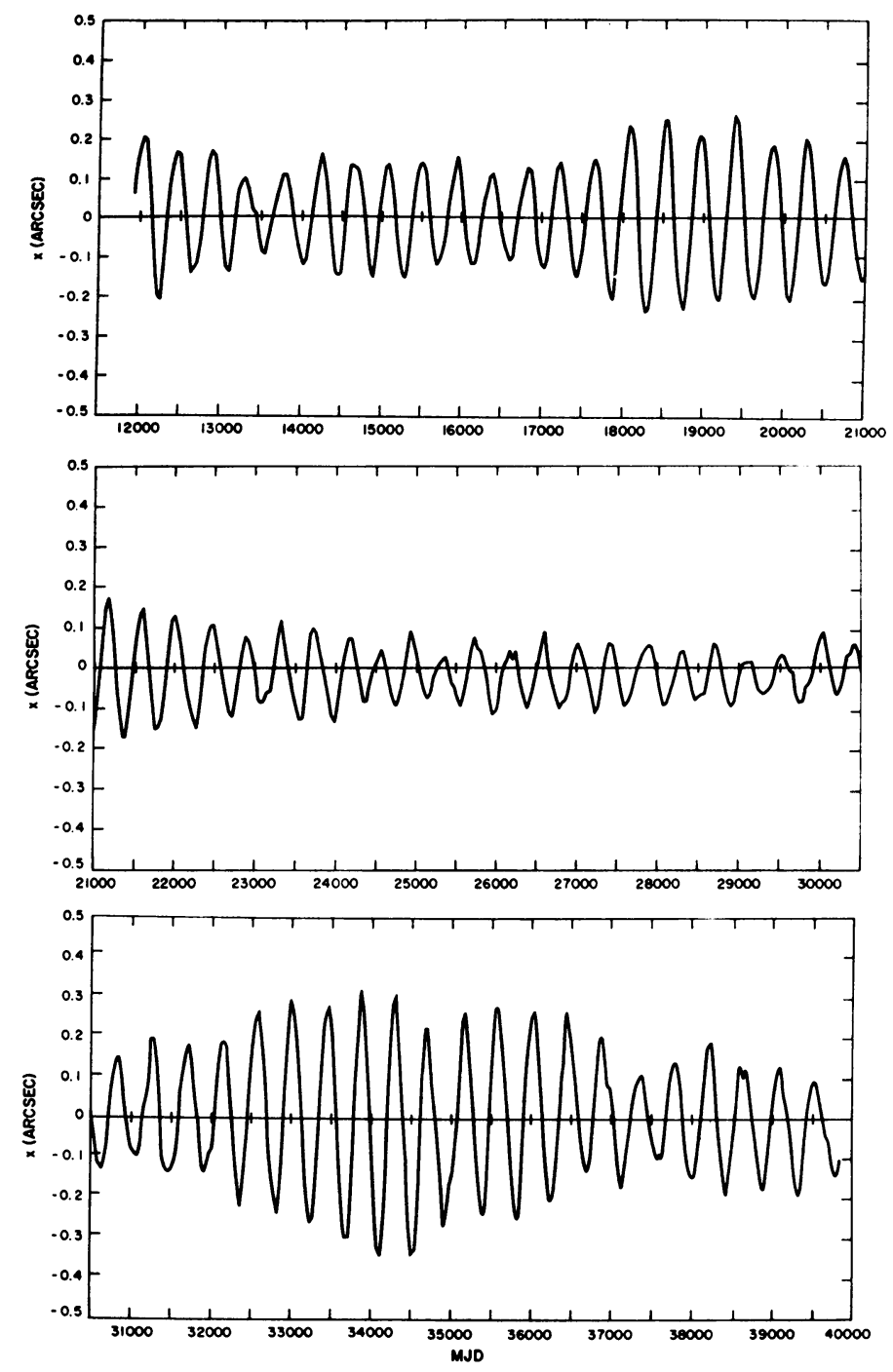

Fig. 2. $x$ component with the 365.25 -day term removed.

Figure 1 shows the cross-correlation function for the $x$ component. There are three well-defined peaks, at 365,428 , and 437.5 days. The first is the annual term. The motion is found to be

$$
\begin{aligned}
& x_{365.25}=-0.0310 \sin \frac{2 \pi}{365.25}\left(t-t_{0}\right)+0 " 0846 \cos \frac{2 \pi}{365.25}\left(t-t_{0}\right), \\
& y_{365.25}=-0 " 0617 \sin \frac{2 \pi}{365.25}\left(t-t_{0}\right)-0 " 0260 \cos \frac{2 \pi}{365.25}\left(t-t_{0}\right),
\end{aligned}
$$


or, given in the same form as Table 9.1 of Munk and MacDonald (1960, p. 91),

$$
\begin{aligned}
& m_{1}=-4.23 \cos \odot-7.96 \sin \odot, \\
& m_{2}=5.76 \cos \odot-3.41 \sin \odot,
\end{aligned}
$$

where the units of $m_{1}$ and $m_{2}$ are 0.01 and the argument is the longitude of the mean sun. The agreement of these results with those of the various authors summarized by Munk and MacDonald is about as good as the agreement of the latter results among themselves. There is no clear choice. Their average is

$$
\begin{aligned}
& m_{1}=-4.35 \cos \odot-7.65 \sin \odot, \\
& m_{2}=6.76 \cos \odot-3.44 \sin \odot .
\end{aligned}
$$

However, this average can be quite misleading since common data were used.

When the annual 365.25-day component given above is removed from the data set, a very important result occurs. The side lobes contained in the original spectrum disappear completely. These lobes, the result of using a finite data set with errors of observation, are not real. Figure 2 is a plot of $x$ with the annual component removed. A phenomenon resembling a beat emerges.

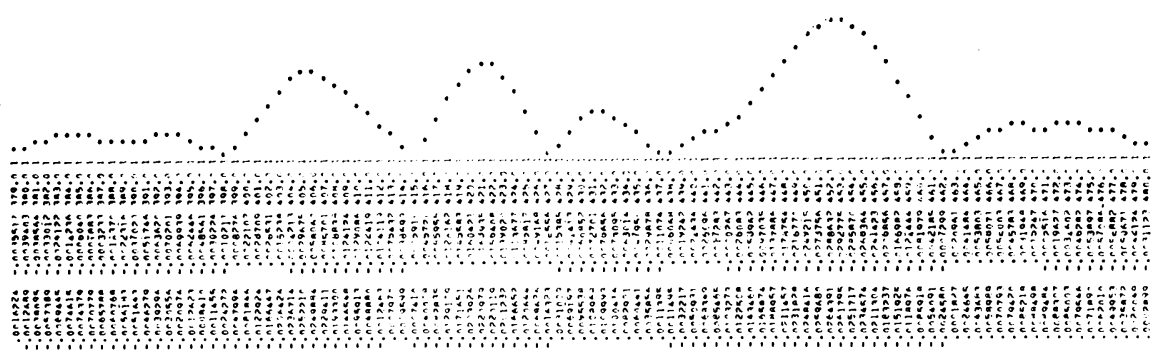

Fig. 3. Residual power spectrum for $x$ with the 365.25-, 428-, and 437.5-day periods removed.

The spectrum between 400 days and 460 days appears to have a continuum in addition to the two peaks. The components of the two peaks are

and

$$
\begin{aligned}
& x_{428}=-0^{\prime \prime} .1179 \sin \left[\frac{2 \pi}{428}\left(t-t_{0}\right)\right]+0.0022 \cos \left[\frac{2 \pi}{428}\left(t-t_{0}\right)\right] \\
& y_{428}=-0.0058 \sin \left[\frac{2 \pi}{428}\left(t-t_{0}\right)\right]-0.1203 \cos \left[\frac{2 \pi}{428}\left(t-t_{0}\right)\right],
\end{aligned}
$$

$$
\begin{aligned}
& x_{437.5}=0.0932 \sin \left[\frac{2 \pi}{437.5}\left(t-t_{0}\right)\right]+0.0075 \cos \left[\frac{2 \pi}{437.5}\left(t-t_{0}\right)\right] \\
& y_{437.5}=-0.0050 \sin \left[\begin{array}{c}
2 \pi \\
437.5
\end{array}\left(t-t_{0}\right)\right]+0.0906 \cos \left[\begin{array}{c}
2 \pi \\
437.5
\end{array}\left(t-t_{0}\right)\right] .
\end{aligned}
$$


After extraction of these components, the remaining spectrum does not essentially reduce to the noise level but has a sum square of 0 ".05. Figure 3 shows the spectrum that remains. Indeed, the residue has the same periodicity but a lower amplitude. From the spectrum analysis, the amplitudes of the three components at 406.5, 421.5, and 452.5 days are

$$
\begin{aligned}
& x_{406.5}=-0.0312 \sin \left[\frac{2 \pi}{406.5}\left(t-t_{0}\right)\right]+0.0007 \cos \left[\frac{2 \pi}{406.5}\left(t-t_{0}\right)\right] \\
& y_{406.5}=-0.0023 \sin \left[\frac{2 \pi}{406.5}\left(t-t_{0}\right)\right]-0^{\prime \prime} 0254 \cos \left[\frac{2 \pi}{406.5}\left(t-t_{0}\right)\right], \\
& x_{421.5}=-0.0210 \sin \left[\frac{2 \pi}{421.5}\left(t-t_{0}\right)\right]+0.0141 \cos \left[\frac{2 \pi}{421.5}\left(t-t_{0}\right)\right] \\
& y_{421.5}=-0.0113 \sin \left[\frac{2 \pi}{421.5}\left(t-t_{0}\right)\right]-0^{\prime \prime} 0166 \cos \left[\frac{2 \pi}{421.5}\left(t-t_{0}\right)\right],
\end{aligned}
$$

and

$$
\begin{aligned}
& x_{452.5}=-0.0228 \sin \left[\frac{2 \pi}{452.5}\left(t-t_{0}\right)\right]-0.0270 \cos \left[\frac{2 \pi}{452.5}\left(t-t_{0}\right)\right] \\
& y_{452.5}=0.0276 \sin \left[\begin{array}{c}
2 \pi \\
452.5
\end{array}\left(t-t_{0}\right)\right]-0.0246 \cos \left[\frac{2 \pi}{452.5}\left(t-t_{0}\right)\right],
\end{aligned}
$$

Figure 4 (See page 23) shows the spectrum with all six components removed, and Figure 5 plots the remaining motion in $x$. The residue seems to have some systematic components; however, this amplitude is less than 0 ". 01 .

These results are in agreement with those of Table II. We can reconcile the continuous-variation model of Melchior by noting that

$$
\sin \alpha+\sin \beta=2 \sin \frac{\alpha+\beta}{2} \cos \frac{\alpha-\beta}{2} .
$$

The two major Chandler peaks would appear to vary in amplitude. To test this, a 10 -yr moving window was computed. Figure 6 gives the amplitude and is in agreement with the analyses of Melchior and of Myerson (1970). In addition, an analysis by Rykhlova (1969) of the long-period pole position resulted in a 40-yr period, nearly the beat period found here. The arguments of Munk and MacDonald appear to assume that the Love number or rigidity varies with time. However, the evidence given here indicates that a two-parameter system would be appropriate and that no temporal change in physical properties is necessary.

The two-frequency interpretation of $Q$ is more favorable, where $Q$ is estimated to be greater than 100 . The dissipation problem is easier than in the one-component model.

The 407-day period arises in the theory of free nutation by Molodensky (1961). This is the period of an elastic earth with a liquid core and no oceans.

Finally, the only substantive argument against this analysis is by Federov and Yatskiv 
(1965), who propose that the motion underwent a $180^{\circ}$ phase change in 1922 . There is no evidence of this, as a plot of the data during that interval will show. If this change in phase is a problem in the reduction of the data, then the annual component should exhibit the same thing, but it does not. The annual component is remarkably constant for all periods.

\section{Analysis of Data from 1846.0 to 1971.0}

The argument by Federov and Yatskiv (1965) prompted the following analysis. We
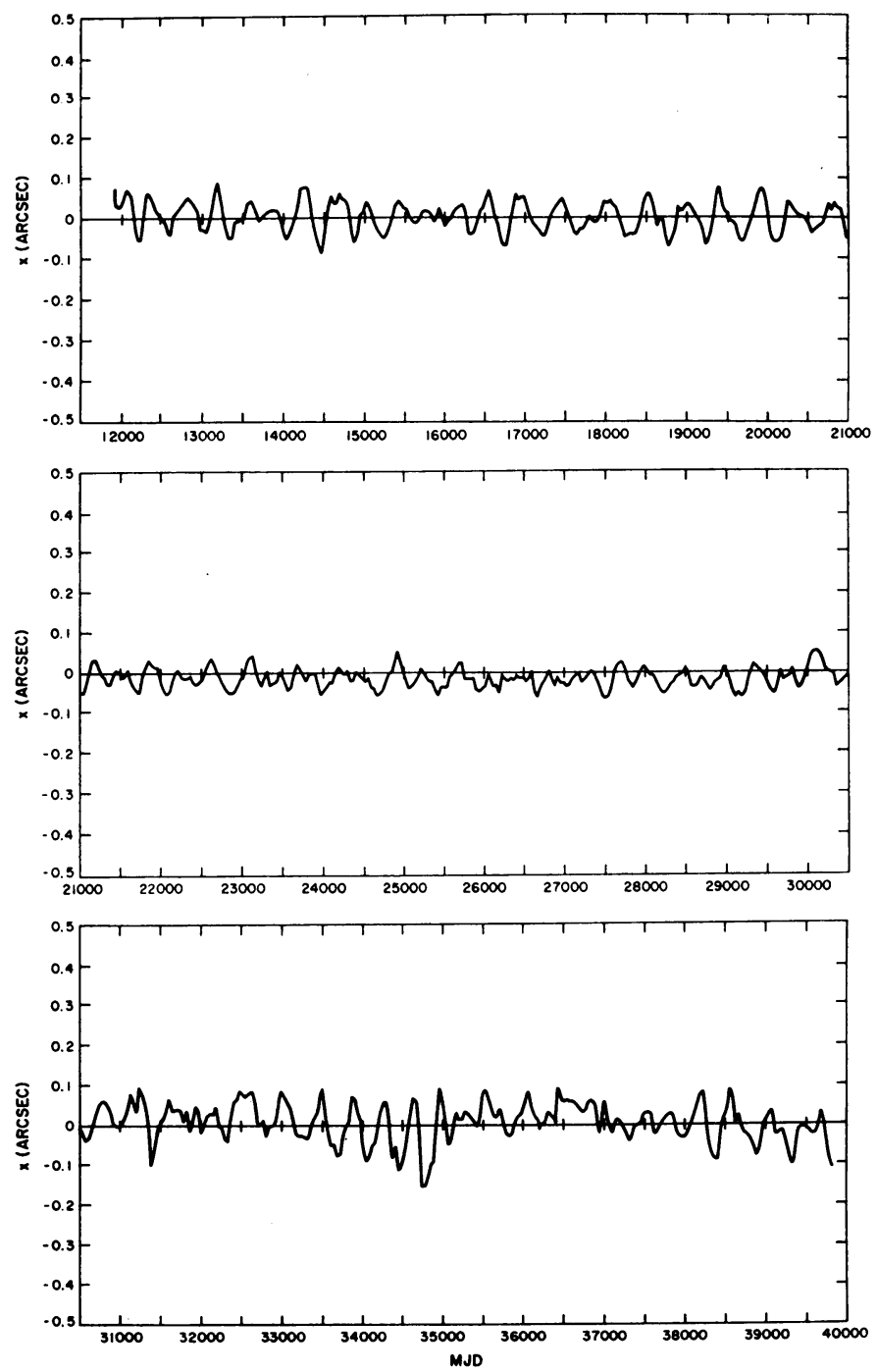

Fig. 5. Residuals with the 365.25-, 428-, 437.5-, 406.5-, 421.5-, and 452.5-day periods removed. 


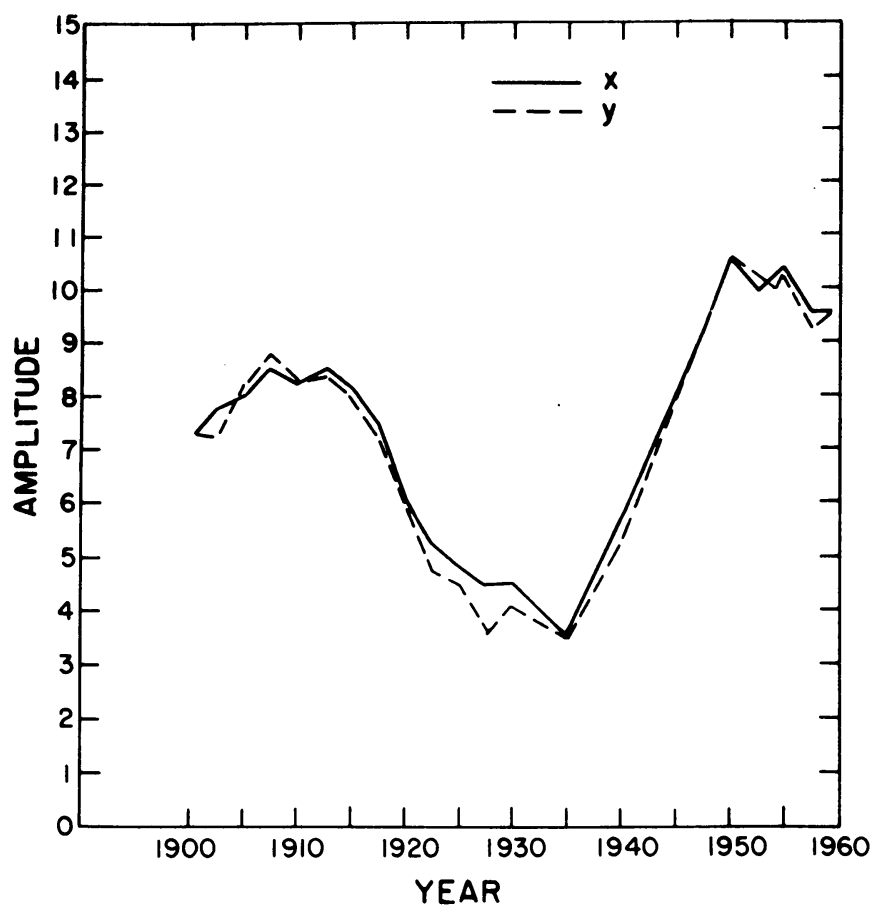

Fig. 6. Amplitude of $x$ from running 10-yr samples.

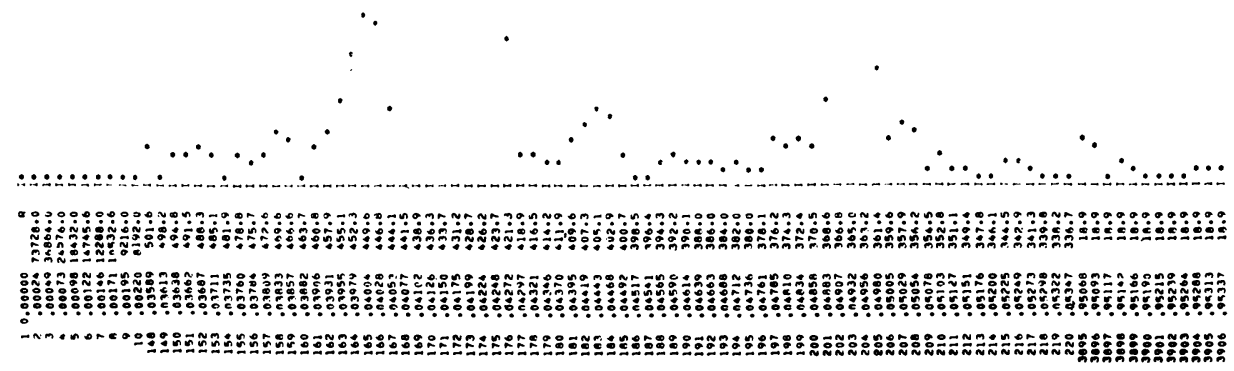

Fig. 7. Discrete Fourier transform of data between 1846.0 and 1971.0 obtained with the use of a Hann filter. 
obtained the data to 1846.0 from Rykhlova (1967), which were sparse. From the harmonic analysis, the amplitude and phase of all components agree. We then combine the two components as $\mathbf{x}=\mathbf{X}+i y$; we gain reliability of the spectrum analysis at the expense of generality. For example, the annual term has a slightly elliptical shape. Treating the data as a single complex function, we assume it is circular.

The data were reduced by use of a 7-yr moving average following Orlov (1942). A three-point Lagrangian interpolation was used to produce 18-day data points for the whole interval. A spectral filter due to Hann was used to sharpen the frequency resolution.

The interval from 1922 to 1970 is too short to resolve the two main frequencies. The data from 1846.0 to 1922 are adequate but much noisier. The full data set exhibits the same frequencies, and the amplitude is plotted in Figure 7. The good agreement of this with Figure 1 is in itself evidence for the validity of the two frequencies. The full data set exhibits the five main frequencies, and the values determined are as follows:

where

$$
\begin{aligned}
\mathbf{x}_{365.25} & =(0.0631-0.0313 i) \exp \left[-2 \pi i\left(\frac{t-t_{0}}{365.25}\right)\right] \\
\mathbf{x}_{428} & =(-0.0199-0.0991 i) \exp \left[-2 \pi i\left(\frac{t-t_{0}}{428}\right)\right] \\
\mathbf{x}_{437.5} & =(0.0048+0.0960 i) \exp \left[-2 \pi i\left(\frac{t-t_{0}}{437.5}\right)\right] \\
\mathbf{x}_{452.5} & =(-0.0163-0.0144 i) \exp \left[-2 \pi i\left(\frac{t-t_{0}}{452.5}\right)\right] \\
\mathbf{x}_{421.5} & =(0.0079-0.0345 i) \exp \left[-2 \pi i\left(\frac{t-t_{0}}{421.5}\right)\right]
\end{aligned}
$$

$$
t_{0}=25887 \text { MJD }
$$

Taking the data set from 1846 to 1922 gives

$$
\begin{aligned}
\mathbf{x}_{365.25} & =(0.0514-0.0364 i) \exp \left[-2 \pi i\left(\frac{t-t_{0}}{365.25}\right)\right] \\
\mathbf{x}_{428} & =(-0.0675-0.0572 i) \exp \left[-2 \pi i\left(\frac{t-t_{0}}{428}\right)\right] \\
\mathbf{x}_{437.5} & =(-0.0764+0.0661 i) \exp \left[-2 \pi i\left(\frac{t-t_{0}}{437.5}\right)\right] .
\end{aligned}
$$

As an example of the constancy, the annual term derived from 1891 to 1970 is

$$
\mathbf{x}_{365.25}=(0.0721-0.0352 i) \exp \left[-2 \pi i\left(\frac{t-t_{0}}{365.25}\right)\right] \text {. }
$$


The agreement is plausible, but the remaining spectrum is quite noisy. The analysis indicates the existence of at least two main frequencies, not using data after 1922 .

We can explore the one-component model further by asking if there is an acceptable exciting function for it. We can write the dynamical model as

$$
(A+\sigma) \dot{\mathbf{x}}-i \mathbf{x}(C-A-\sigma)=\mathbf{f}(t)
$$

where

$$
\boldsymbol{\sigma}=\mathbf{k} \frac{\omega_{e}^{2} a_{e}^{5}}{3 G}
$$

and the complex Love number $\mathbf{k}$ (Munk and MacDonald, 1960, p. 153) is

$$
\mathbf{k}=k\left(1-i \frac{\mu}{2 Q}\right)
$$

The rigidity of the Earth $\mu=2.26$ in these units, $k \cong 0.28$, and $Q$ is to be determined.

The Fourier transform of Equation (1) is

$$
\mathbf{X}(\xi)[\xi(A+\boldsymbol{\sigma})-(C-A-\boldsymbol{\sigma})]=\mathbf{F} .
$$

If $f(t)$ is a set of unit impulses - i.e.,

$$
\mathbf{f}(t)=\sum_{j=1} \mathbf{D}_{j} \delta\left(t-T_{j}\right)
$$

then

$$
\mathbf{F}=\sum_{j=1} D_{j} \exp \left(-2 \pi i \xi T_{j}\right)
$$

i.e., harmonic oscillation in $\xi$ space. We have $\mathbf{X}(\xi)$ by transforming the observed data and can compute the left-hand side, given $k$ and $Q$. We then take the Fourier transform of Equation (2). The poles of the transform should correspond to times of excitation.

It is clear from Equation (1) that unless $Q>20$ the complex $\mathbf{k}$ cannot annihilate both frequencies when used in Equation (2), which is another way of saying that the wide spectrum requires a low $Q$. With such a low $Q$, if Federov and Yatskiv's conjecture is correct, the excitation must have occurred soon before the postulated change in phase. No such event is evident in the preliminary analysis.

The excitation function modelled by Equation (3) cannot be recovered. The alternative is a continuous pumping of energy into the motion. Such a mechanism was proposed by Colombo and Shapiro (1968). With the larger $Q$, the amount of excitation needed is reduced.

\section{Discussion}

(1) Analysis of polar-motion data for $125 \mathrm{yr}$ indicates that a model with at least two degrees of freedom is necessary for a determination of the polar motion. The natural frequencies of the earth are 428 and 437.5 .

(2) Such a model overcomes the dissipation problem and indicates $Q>100$.

(3) The annual motion is well determined. 
(4) The exciting mechanism for the Chandler motion has not yet been inferred from the data, and further analysis is in order.

(5) New techniques for determining pole position more accurately will not improve this analysis until many years of data become available. Therefore, revision of the historical data would be valuable.

Note added in proof: I have revised this analysis in two ways. First, the pole positions are given by Federov et al. (1972). Second, a least-squares adjustment process has been developed, based on the analytical form for the DFT, which determines the complex amplitude and frequency of the data. The essential results are unchanged. The annual and two Chandler components are as follows:

$$
\begin{aligned}
\mathbf{x}_{365.248}=\left(-0^{\prime \prime} 03389-\right. & 0.07419 i) \times \\
& \times \exp \left[2 \pi(-0.0000227-0.0985632 i)\left(\frac{t-t_{0}}{36}\right)\right] \\
\mathbf{x}_{437.196}=(-0.05452+ & 0.02597 i) \times \\
& \quad \times \exp \left[2 \pi(0.0001502-0.0823430 i)\left(\frac{t-t_{0}}{36}\right)\right] \\
\mathbf{x}_{426.670=}=\left(0^{\prime \prime} 01423-0.1019 i\right) \times & \quad \times \exp \left[2 \pi(0.0000179-0.0843744 i)\left(\frac{t-t_{0}}{36}\right)\right], \\
t_{0}= & 11367 \text { MJD. }
\end{aligned}
$$

Additional terms were found:

$$
\begin{aligned}
\mathbf{x}_{452.890}=(-0.02502- & 003465 i) \times \\
& \times \exp \left[2 \pi(-0.0000175-0.0794894 i)\left(\frac{t-t_{0}}{36}\right)\right]
\end{aligned}
$$

and

$$
\begin{aligned}
\mathbf{x}_{406.44}=(-0.01642+0 & 00996 i) \times \\
& \times \exp \left[2 \pi(0.0000865-0.0885732 i)\left(\frac{t-t_{0}}{36}\right)\right] .
\end{aligned}
$$

However, the 421-day term is not recovered. These new data are superior to those used in the previous analysis, especially in the noise, which is significantly reduced.

\section{References}

Carnera, L.: 1948, Bull. Géodés. No. 10, 269.

Carnera, L.: 1949, Bull. Géodés. No. 13, 267.

Cecchini, G.: 1951, Bull. Géodés. No. 21, 275

Cecchini, G.: 1962, Bureau Central des Telegrammes Astronomiques Circulaire No. 1804 of August 15

(coordinates supplied by Cecchini, Torino). 
Chandler, S.: 1891, Astron. J. 11, 83.

Colombo, G. and Shapiro, I. I.: 1968, Nature 217, 156.

Coulomb, J. and Jobert, G.: 1963, The Physical Constitution of the Earth, (transl. by A. E. M. Nairn), Hafner, Publ. Co., New York.

Federov, Ye. P.: 1963, Nutation and Forced Motion of the Earth's Pole, (transl. by B. S. Jeffreys), Pergamon, New York.

Federov, E. P., Korsun, A. A., Major, S. P., Panchenko, N. T., Tarady, V. K., and Yatskiv, Ya. A.: 1972, this volume, p. 12.

Federov, E. P. and Yatskiv, Ya. S.: 1965, Soviet Astron. 8, 608.

Gutenberg, G.: 1959, Physics of the Earth's Interior, Int. Geophys. Ser., 1, Academic Press, New York. International Astronomical Union, Commission No. 19: 1955, Trans. Int. Astron. Un. IX, 277.

International Polar Motion Service: 1971, Monthly Notices IPMS,

Jeffreys, H.: 1940, Monthly Notices Roy. Astron. Soc. 100, 139.

Jeffreys, H.: 1968, Monthly Notices Roy. Astron. Soc. 141, 255.

Jeffreys, H.: 1970, The Earth, 5 th ed., Cambridge Univ. Press, England.

Kaula, W. M.: 1968, An Introduction to Planetary Physics: The Terrestrial Planets, John Wiley \& Sons, Inc., New York.

Markowitz, W.: 1968, in Wm. Markowitz and B. Guinot (eds.), 'Continental Drift, Secular Motion of the Pole, and Rotation of the Earth', IAU Symp. 32, 25.

Melchior, P. J.: 1955, Rc. Accad. Lincea Roma, Ser. VIII, XIX (fasc. 3-4), 137.

Melchior, P. J.: 1957, in L. H. Ahrens et al. (eds), Physics and Chemistry of the Earth, Pergamon, New York, vol. 2, p. 212.

Molodensky, M. S.: 1961, The theory of nutation and diurnal earth tides, IV Symp. Int. Marees Terre Comm. Obs. Roy. Belg., No. 188, Studia Géophys., No. 58, 25.

Munk, W. H. and MacDonald, G. J. F.: 1960, The Rotation of the Earth, Cambridge Univ. Press, England.

Myerson, R. J.: 1970, J. Geophys. Res. 75, 6612.

Nesterov, V. V. and Rykhlova, L. V.: 1970, Soviet Astron. 14, 340.

Nicolini, T.: 1968, in Wm. Markowitz and B. Guinot (eds.), 'Continental Drift, Secular Motion of the Pole, and Rotation of the Earth', IAU Symp. 32, 101.

Orlov, A.: 1942, Compt. Rend. Acad. Sci. U.R.S.S. 37, 264.

Rudnick, P.: 1956, Am. Geophys. Un. Trans. 37, 137.

Rykhlova, L. V.: 1967, Soviet Astron. 12, 898.

Rykhlova, L. V.: 1969, Soviet Astron. 13, 544.

Walker, A. M. and Young, A.: 1957, Monthly Notices Roy. Astron. Soc. 117, 119.

Yaskov, V. Ya.: 1965, Soviet Astron. 8, 605.

Yumi, S.: 1964, Ann. Rep. IPMS for 1962, International Polar Motion Service, Mizusawa, Japan. Yumi, S.: 1965, Ann. Rep. IPMS for 1963, International Polar Motion Service, Mizusawa, Japan. Yumi, S.: 1966, Ann. Rep. IPMS for 1964, International Polar Motion Service, Mizusawa, Japan.

\section{DISCUSSION}

W. Fricke: What basic observational data from 1846 to about 1890 were used in your analysis? I have so far doubted the reliability of conslusions on polar motion based on observations of that period of time.

E. M. Gaposchkin: The data were obtained from Pulkovo, Greenwich and Washington. They were reduced by Madam Rykhlova, 1967. 